Clearly the left hand members of these conditions are now no longer independent of the displacements $x_{i}$, and the question of whether the new allele will increase now depends upon the nature of the disturbance which is created among the old alleles by the introduction of the new gene. For instance, if the new gene is introduced into the population at the expense of allele $A_{1}$ only, while the frequencies of $A_{2}, A_{3}, \ldots, A_{n}$ remain unchanged, then condition (9) reduces to

$$
\sum_{j=1}^{n}\left(a_{1 j}-a_{n+1}, j\right) p_{j}<0
$$

and this condition may clearly be interpreted in a very simple fashion.

\title{
REFERENCES
}

AtKinson, F. V., WAtrerson, G. A., AND Moran, P. A. P. 1960. A matrix inequality. Quart. F. Math. Oxford (2), II, 137-140.

BODMRR, W. F., AND PARSONS, P. A. 1960. The initial progress of new genes with various genetic systems. Heredity, 15, 283-299.

FISHER, R. A. 1922. On the dominance ratio. Proc. Roy. Soc. Edinb., 42, 321-341.

HALDANE, J. B. S. 1957. The conditions for co-adaptation in polymorphism for inversions. F. Genet., 55, $218-225$.

KINGMan, J. F. C. 1961. On an inequality in partial averages. Quart. 7. Math Oxford (2), 12, 78-80.

Mandel, S. P. H. 1959. The stability of a multiple allelic system. Heredity, 13, 289-302.

MULhollaND, H. P., AND SMiTH, C. A. B. 1959. An inequality arising in genetical theory. Am. Math. Month., 66, 673-683.

OWEN, A. R. G. I954. Balanced polymorphism of a multiple allelic series. Caryologia, Supp., 6, 1240-1241.

SCHEUER, P. A. G., AND MANDEL, S. P. H. 1959. An inequality in population genetics.

Heredity, I3, 519-524.

\section{CYTOLOGICAL STUDIES IN THE GRAMINEA (II)}

D. N. SINGH and M. B. E. GODWARD

Botany Department, Queen Mary College, London

This second list of chromosome numbers continues that published earlier by Singh and Godward (I960), which included data utilised by Clayton (1962) in support of the separation of the genera Tetrapogon and Chloris. It is accordingly numbered table 2 to follow table $I$ of the previous paper. 
TABLE 2

\begin{tabular}{|c|c|c|c|c|c|}
\hline \multirow{2}{*}{\multicolumn{3}{|c|}{ Name }} & \multicolumn{2}{|c|}{$\begin{array}{l}\text { Chromosome } \\
\text { counts }\end{array}$} & \multirow{2}{*}{ Source } \\
\hline & & & $n$ & $2 n$ & \\
\hline \multicolumn{6}{|c|}{ TRIBE.-PANICE $A E$} \\
\hline$x=9$ & $\begin{array}{l}\text { PANICUM } \\
\text { miliaceum L. } \\
\text { var. contractum Alef. }\end{array}$ & & $\cdots$ & $\begin{array}{l}3^{6} \\
3^{6}\end{array}$ & $\begin{array}{l}\text { Patna, India } \\
\text { Zak. Bot. Ogolney, Poland } 1\end{array}$ \\
\hline \multicolumn{6}{|c|}{ TRIBE.-SPOROBOLEE } \\
\hline$x=9$ & $\begin{array}{l}\text { SPOROBOLUS } \\
\text { africanus (Poir.) }\end{array}$ & . & $\cdots$ & 36 & Royal Botanic Gardens, Kew \\
\hline \multicolumn{6}{|c|}{ TRIBE.-CHLORIDE $\mathscr{E}$} \\
\hline$x=\mathrm{I0}$ & $\begin{array}{l}\text { CHLORIS } \\
\text { gayana Kunth }\end{array}$ & · & $\cdots$ & $20,20,4^{\circ}$ & Kitale, Kenya \\
\hline \multicolumn{6}{|c|}{ TrIBe. $-F E S T L C E A E$} \\
\hline$x=7$ & $\begin{array}{l}\text { CATAPODIUM } \\
\text { rigidum (L.) C. E. Hubbard }\end{array}$ & & $\cdots$ & $14+I$ to 5 & Royal Botanic Gardens, Kew \\
\hline \multicolumn{6}{|c|}{ Tribe.-BROME $E$} \\
\hline $\begin{array}{l}x=7 \\
x=7\end{array}$ & $\begin{array}{l}\text { BROMUS } \\
\text { coloratus Steud. } \\
\text { VULPIA } \\
\text { megalura (Nutt.) Rydberg }\end{array}$ & $\cdot$ & $\begin{array}{l}\cdots \\
\cdots\end{array}$ & $\begin{array}{l}42 \\
63\end{array}$ & $\begin{array}{l}\text { Royal Botanic Gardens, Kew } \\
\text { Royal Botanic Gardens, Kew }\end{array}$ \\
\hline \multicolumn{6}{|c|}{ TRIBE.-MELICE $E$} \\
\hline$x=6$ & $\begin{array}{l}\text { MELICA } \\
\text { altissima Linn. . } \\
\text { transsilvanica Schur. . }\end{array}$ & $\cdot$ & $\begin{array}{l}\cdots \\
\cdots\end{array}$ & $\begin{array}{l}\text { I } 8 \\
\text { I } 8\end{array}$ & $\begin{array}{l}\text { Royal Botanic Gardens, Kew } \\
\text { Royal Botanic Gardens, Kew }\end{array}$ \\
\hline \multicolumn{6}{|c|}{ TrIBE. $-A V E N E A E$} \\
\hline $\begin{array}{l}x=7 \\
x=7\end{array}$ & $\begin{array}{l}\text { TRISETUM } \\
\text { paniceum Pers. } \\
\text { KGELERIA } \\
\text { alpicola Gren. \& Godr. } \\
\text { phleoides Pers. . }\end{array}$ & $\dot{r}$ & $\begin{array}{l}\cdots \\
\cdots\end{array}$ & $\begin{array}{c}4^{2}+\mathrm{IB} \\
28\end{array}$ & $\begin{array}{l}\text { Royal Botanic Gardens, Kew } \\
\text { Royal Botanic Gardens, Kew } \\
\text { Royal Botanic Gardens, Kew }\end{array}$ \\
\hline
\end{tabular}

1 Seeds received through Chelsea Physic Garden, London. 


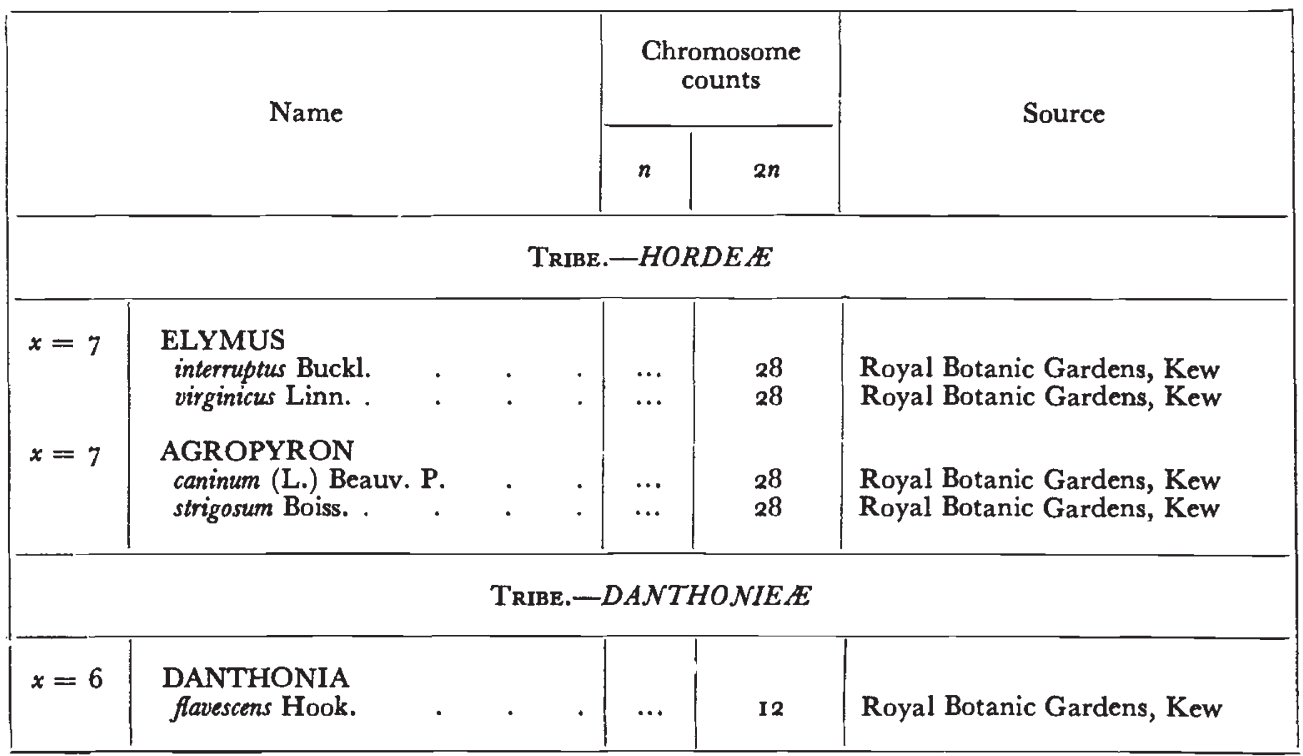

\section{REFERENCES}

SINGH, D. N., AND GODWARD, M. B. E. Ig6o. Cytological studies in the Gramineæ.

Heredity, 15, 193-197.

CLAYTON, W. D. 1962. Studies in the Graminex II. Kew Bull., 16, 248-250.

\section{MUTUAL REDUCTION OF DOMINANCE INVOLVING THREE LOCI IN MUS MUSCULUS}

JEAN A. H. TATCHELL

Department of Genetics, University of Cambridge

Received 3o.v.63

When the heterozygote becomes phenotypically distinct from the dominant homozygote due to the presence of a mutant gene at a second locus, reduction of the dominance of the first gene can be said to have taken place. This phenomenon was first noticed in mice in I9I I by Durham, who observed that $B B$ became distinct from $B b$ when on a pink-eye $(p p)$ background, and again in 193 I by Snell. However, it was not until 1953 that Wallace showed this reduction to be mutual, $P P$ and $P p$ being distinguishable from each other when on a $b b$ background, thus giving rise to six distinct phenotypes instead of the expected four (table $r$ ).

Goodwins (1958) further showed that on a dilute $(d d)$ background the 